\title{
Generalized fractional Hilfer integral and derivative
}

\author{
Juan E. Nápoles Valdés* \\ Facultad de Ciencias Exactas y Naturales y Agrimensura, Universidad Nacional del Nordeste, Av. Libertad 5450, Corrientes, Argentina \\ Facultad Regional Resistencia, Universidad Tecnologica Nacional, French 414, Resistencia, Chaco, Argentina
}

(Received: 5 October 2020. Received in revised form: 21 December 2020. Accepted: 28 December 2020. Published online: 31 December 2020.)

(c) 2020 the author. This is an open access article under the CC BY (International 4.0) license (www.creativecommons.org/licenses/by/4.0/).

\begin{abstract}
In this paper, the author introduces the generalized fractional Hilfer integral and derivative of order $\mu$, which are natural extensions of some recently defined fractional operators.
\end{abstract}

Keywords: generalized fractional Hilfer integral; fractional calculus.

2020 Mathematics Subject Classification: 26A33.

\section{Introduction}

Fractional calculus, a branch of mathematics, is focused on the study and applications of the differential and integral operators of non-integer order. Although the fractional calculus is as old as the classical calculus, it has become one of the most developed areas of mathematics only in the last 40 years, not only because of the exponential growth of the number of publications in this area, but also due to its different applications and its overlapping with other areas of mathematics. This area has been developed intensively in recent years and it has found multiple applications in various fields. The classical results were basically extended in two fundamental directions: Riemann-Liouville fractional derivative and Caputo fractional derivative.

As a result of the progress made in this area, numerous fractional (global) and generalized (local) operators have been appeared. These new operators give researchers the possibility to choose the one that suits best with the problem they investigate. Readers can consult the paper [2] where some reasons are given to justify the appearance of these new operators and where the applications and theoretical developments of these operators are discussed. These operators, developed by many mathematicians with a hardly specific formulation, include the Riemann-Liouville (RL), Weyl, Erdelyi-Kober and Hadamard integrals, and the fractional operators of Liouville and Katugampola. Many authors have even introduced new fractional operators generated from the general local differential operators. In this direction, a generalized local derivative was defined in [19], which generalizes both the conformable and non-conformable derivatives and that is the basis for the generalized integral operator proposed in [7], which contains as a particular case the fractional integral of RiemannLiouville (see [31]). In fact, these new operators require a classification as they can cause confusion in researchers. Baleanu and Fernandez [3] gave a fairly complete classification of these fractional and generalized operators together with abundant information and references. For a more complete review, the readers are referred to Chapter 1 of [1], where a history of differential operators (both local and global) from Newton to Caputo is presented and where the qualitative differences between the operators are shown. Section 1.4 of [1] contains some conclusions that we want to highlight: "Therefore, we can conclude that the Riemann-Liouville and Caputo operators are not derivatives and, therefore, they are not fractional derivatives, but fractional operators. We agree with the result [27] that the local fractional operator is not a fractional derivative" (see p.24 in [1]).

In this work, we present a new definition of the $k$-generalized fractional derivative of the Hilfer type, and we study its fundamental properties. We also present a particular case with a kernel defined in terms of the sigmoid function.

The gamma function $\Gamma$ (see $[21,24,28,29]$ ) and $k$-generalized gamma function $\Gamma_{k}$ (see [6]) are defined as:

$$
\begin{aligned}
& \Gamma(z)=\int_{0}^{\infty} t^{z-1} e^{-t} \mathrm{~d} t, \quad \Re(z)>0, \\
& \Gamma_{k}(z)=\int_{0}^{\infty} t^{z-1} e^{-t^{k} / k} \mathrm{~d} t, \quad k>0 .
\end{aligned}
$$

*E-mail address: jnapoles@exa.unne.edu.ar 
It is clear that $\Gamma_{k}(z)=(k)^{\frac{z}{k}-1} \Gamma\left(\frac{z}{k}\right), \Gamma_{k}(z+k)=z \Gamma_{k}(z)$ and if $k \rightarrow 1$ then $\Gamma_{k}(z) \rightarrow \Gamma(z)$.

Next, we present several definitions of the fractional operators, with their properties and notation which we use later (further details can be found in [15]).

Let $-\infty<a<b<+\infty$. Denote by $C[a, b], A C[a, b]$ and $C^{n}[a, b]$ the spaces of continuous, absolutely continuous and $n$ times continuously differentiable functions on $[a, b]$, respectively. For $p \geq 1$, denote by $L^{p}[a, b]$ the spaces of Lebesgue integrable functions on $[a, b]$. Let us first define the classic Riemann-Liouville fractional integrals.

Definition 1.1. Let $f \in L_{1}[a, b]$. Then the Riemann-Liouville fractional integrals (right and left respectively) of order $\mu \in \mathbb{C}$, $\Re(\mu)>0$, are defined by

$$
\begin{aligned}
& { }^{\mu} J_{a^{+}} f(x)=\frac{1}{\Gamma(\mu)} \int_{a}^{x}(x-t)^{\mu-1} f(t) d t, \quad x>a \\
& { }^{\mu} J_{b^{-}} f(x)=\frac{1}{\Gamma(\mu)} \int_{x}^{b}(t-x)^{\mu-1} f(t) d t, \quad x<b .
\end{aligned}
$$

Definition 1.2. Let $\mu$ be a real number satisfying the inequality $m-1<\mu \leq m$ where $m$ is a positive integer. The left and right fractional Riemann-Liouville derivatives of order $\mu$ are defined as

$$
\begin{array}{ll}
D_{+}^{\mu} f(x)=\frac{d^{m}}{d x^{m}}\left[{ }^{m-\mu} J_{a+} f(t)\right], & m-1<\mu \leq m, \\
D_{-}^{\mu} f(x)=\frac{d^{m}}{d x^{m}}\left[{ }^{m-\mu} J_{b-} f(t)\right], & m-1<\mu \leq m .
\end{array}
$$

The above equation regarding $D_{+}^{\mu} f(x)$ is equivalent to

$$
D_{+}^{\mu} f(x)=\left\{\begin{array}{l}
\frac{d^{m}}{d x^{m}}\left[\frac{1}{\Gamma(\mu)} \int_{a}^{x} \frac{f(t) d t}{(x-t)^{1+\mu-m}}\right], \quad m-1<\mu \leq m, \\
\frac{d^{m} f(x)}{d x^{m}}, \quad \mu=m,
\end{array}\right.
$$

and similarly one can write an alternative expression for $D_{-}^{\mu} f(x)$.

Remark 1.1. Another way to define these fractional derivatives is to consider them as the left (and right) inverse of the $k$-generalized fractional Riemann-Liouville integral with general kernel of order $\mu$, that is

$$
\begin{aligned}
& D_{+}^{\mu}\left[{ }^{m-\mu} J_{a+}\right]=I d, \\
& D_{-}^{\mu}\left[{ }^{m-\mu} J_{b-}\right]=I d,
\end{aligned}
$$

with $m-1<\mu \leq m$.

Remark 1.2. The Riemann-Liouville integral can also be expressed as ${ }^{\mu} J_{a+} f(x)=\left(f * K_{\mu}\right)(x)$, with $K_{\mu}(x)=\frac{x^{\mu-1}}{\Gamma(\mu)}$ the kernel of convolution product.

In 1967, Michele Caputo gave a new definition of fractional derivative in [5] with an interesting property: when solving differential equations using Caputo's definition, it is not necessary to define the fractional order initial conditions. This derivative has been used in the study of many applied problems (see [4]).

Definition 1.3. The Caputo derivative of a differentiable function $f$ of order $\mu, 0<\mu<1$, is defined as

$$
{ }^{C} D^{\mu} f(t)=\frac{1}{\Gamma(1-\mu)} \int_{0}^{t} \frac{f^{\prime}(s)}{(t-s)^{\mu}} d s .
$$

Remark 1.3. Keeping in mind Definition 1.2, we mention here one of the advantages of the Riemann-Liouville (RL) derivative over the Caputo $(C)$ derivative. It is an obvious fact that to calculate the $R L$ derivative of order $\mu$ with $\mu \in(0,1)$, it is not necessary for the function to have an ordinary first derivative, a requirement that the $C$ derivative does have (see Definition 1.3). Thus, there are functions that do not have first-order derivatives but they can have fractional derivatives of all orders less than one in the Riemann-Liouville sense.

Next, we present the definition of the Riemann-Liouville fractional derivative with respect to another function $\psi$, which is directly related to the Riemann-Liouville fractional derivative (see [15]). 
Definition 1.4. Let $\psi(u)>0$ be an increasing function on $(a, b]$ and $\psi^{\prime}(u)>0$ be continuous on (a,b), and $\mu>0$, where $a, b \in \mathbb{R}$. The $\psi$-fractional integrals (left-sided and right-sided) of order $\mu$ are defined by

$$
\begin{aligned}
& D_{a+}^{\mu ; \psi} f(x)=\left[\frac{1}{\psi^{\prime}(x)} \frac{d}{d x}\right]^{n} I_{a+}^{n-\mu ; \psi} f(x)=\frac{1}{\Gamma(n-\mu)}\left[\frac{1}{\psi^{\prime}(x)} \frac{d}{d x}\right]^{n} \int_{a}^{x} \frac{\psi^{\prime}(t) f(t) d t}{(\psi(x)-\psi(t))^{\mu+1-n}} \\
& D_{b-}^{\mu ; \psi} f(x)=\left[-\frac{1}{\psi^{\prime}(x)} \frac{d}{d x}\right]^{n} I_{b-}^{n-\mu ; \psi} f(x)=\frac{1}{\Gamma(n-\mu)}\left[-\frac{1}{\psi^{\prime}(x)} \frac{d}{d x}\right]^{n} \int_{a}^{x} \frac{\psi^{\prime}(t) f(t) d t}{(\psi(t)-\psi(x))^{\mu+1-n}}
\end{aligned}
$$

with $n=[\mu]+1$.

Typically, fractional derivatives are presented through a combination of integer derivatives, derivatives, and fractional integrals. Hilfer (see [10-12]) gives a definition that is an "intermediate" point of the classical fractional derivatives: Riemann-Liouville and Caputo.

Definition 1.5. The Hilfer fractional derivative of order $\mu, 0<\mu<1$, and type $\beta, 0 \leq \beta \leq 1$, with respect to $x$ is defined by

$$
D_{a+}^{\mu, \beta} f(x)=\left({ }^{1-\mu} J_{a+}^{\beta} \frac{d}{d x}\left({ }^{1-\mu} J_{a+}^{1-\beta} f\right)\right)(x),
$$

for the functions for which the expression on the right hand side exists.

Remark 1.4. In Definition 1.5, if we take $\beta=0$ then we have the Riemann-Liouville derivative (see Definition 1.1) and the choice $\beta=1$ gives us the Caputo derivative (see Definition 1.3).

In order to obtain a more general derivative than the one proposed by Hilfer, Hilfer et al. [13] proposed the following generalized Riemann-Liouville fractional derivative, which is associated with the Riemann-Liouville fractional integral.

Definition 1.6. For $n \in \mathbb{N}$, let $\mu, \beta \in \mathbb{R}$ be the numbers satisfying the inequalities $n-1<\mu \leq n$ and $0 \leq \beta \leq 1$. The Hilfer fractional derivative of order $\mu$ and type $\beta$ with respect to $x$ is defined by

$$
{ }^{n} D_{a+}^{\mu, \beta} f(x)=\left({ }^{n-\mu} J_{a+}^{\beta} \frac{d}{d x}\left({ }^{n-\mu} J_{a+}^{1-\beta} f\right)\right)(x),
$$

for the functions for which the expression on the right hand side exists.

Below is a generalization of the Hilfer derivative specified in Definition 1.5. We call this generalization as the $\psi$ fractional integral and derivative.

Definition 1.7. Let $\psi(u)>0$ be an increasing function on $(a, b]$ and $\psi^{\prime}(u)>0$ be a continuous function on $(a, b)$, and take $\mu>0$, where $a, b \in \mathbb{R}$. The $\psi$-fractional integrals (left-sided and right-sided) of order $\mu$ of a function $f$ defined as follows

$$
\begin{aligned}
& { }^{H} D_{a+}^{\mu, \beta ; \psi} f(x)=I_{a+}^{\beta(n-\mu) ; \psi}\left[\frac{1}{\psi^{\prime}(x)} \frac{d}{d x}\right]^{n} I_{a+}^{(1-\beta)(n-\mu) ; \psi} f(x), \\
& { }^{H} D_{b-}^{\mu, \beta ; \psi} f(x)=I_{b-}^{\beta(n-\mu) ; \psi}\left[-\frac{1}{\psi^{\prime}(x)} \frac{d}{d x}\right]^{n} I_{b-}^{(1-\beta)(n-\mu) ; \psi} f(x),
\end{aligned}
$$

with $n=[\mu]+1$.

The above derivatives can be written in the following form:

$$
\begin{aligned}
& { }^{H} D_{a+}^{\mu, \beta ; \psi} f(x)=I_{a+}^{\mu-\mu ; \psi} D_{a+}^{\mu ; \psi} f(x), \\
& { }^{H} D_{b-}^{\mu, \beta ; \psi} f(x)=I_{b-}^{\mu-\mu ; \psi} D_{b-}^{\mu ; \psi} f(x),
\end{aligned}
$$

with $\mu=\mu+\beta(n-\mu)$ and

$$
\begin{aligned}
& I_{a+}^{\mu ; \psi} f(x)=\frac{1}{\Gamma(\mu)} \int_{a}^{x} \frac{\psi^{\prime}(t) f(t) d t}{(\psi(x)-\psi(t))^{1-\mu}} \\
& I_{b-}^{\mu ; \psi} f(x)=\frac{1}{\Gamma(\mu)} \int_{x}^{b} \frac{\psi^{\prime}(t) f(t) d t}{(\psi(x)-\psi(t))^{1-\mu}} .
\end{aligned}
$$




\section{On the $k$-generalized $\psi$-Hilfer integral}

We are now able to define the $k$-generalized $\psi$-Hilfer integral as follows.

Definition 2.1. Let $\psi(u)>0$ be an increasing function on $(a, b]$ and $\psi^{\prime}(u)>0$ be continuous on $(a, b)$ and $\mu>0$, where $a, b \in \mathbb{R}$. The generalized $\psi$-fractional integrals (left-sided and right-sided) of order $\mu$ are defined by

$$
\begin{aligned}
& I_{G, a+}^{\mu, k ; \psi} f(x)=\frac{1}{k \Gamma_{k}(\mu)} \int_{a}^{x} \frac{\psi^{\prime}(t) f(t) d t}{G\left(\psi(x)-\psi(t), \frac{\mu}{k}\right)}, \\
& I_{G, b-}^{\mu, k ; \psi} f(x)=\frac{1}{k \Gamma_{k}(\mu)} \int_{x}^{b} \frac{\psi^{\prime}(t) f(t) d t}{G\left(\psi(t)-\psi(x), \frac{\mu}{k}\right)},
\end{aligned}
$$

with $k>0$, and $G(z, \mu) \in A C\left[a_{1}, a_{2}\right]$.

We can also define generalized derivatives as follows.

Definition 2.2. Let $\psi(u)>0$ be an increasing function on $(a, b]$, with $a, b \in \mathbb{R}$, and $\psi^{\prime}(u)>0$ be continuous on (a,b), and take $\mu>0$ and $G(z, \mu) \in A C\left[a_{1}, a_{2}\right]$. The generalized $\psi$-fractional derivative (left-sided and right-sided) of order $\mu$ are defined by

$$
\begin{aligned}
& { }^{H} D_{G, a+}^{\mu, \beta ; \psi} f(x)=I_{G, a+}^{\beta(n-\mu) ; \psi}\left[\frac{1}{\psi^{\prime}(x)} \frac{d}{d x}\right]^{n} I_{G, a+}^{(1-\beta)(n-\mu) ; \psi} f(x), \\
& { }^{H} D_{G, b-}^{\mu, \beta ; \psi} f(x)=I_{G, b-}^{\beta(n-\mu) ; \psi}\left[-\frac{1}{\psi^{\prime}(x)} \frac{d}{d x}\right]^{n} I_{G, b-}^{(1-\beta)(n-\mu) ; \psi} f(x),
\end{aligned}
$$

with $n=[\mu]+1$.

Remark 2.1. Several existing integral operators are particular cases of Definition 2.1.

1. In Definition 2.1, if we make $k=1, G(z, \mu)=z^{1-\mu}$ and $\psi^{\prime}(t)=1$ then we obtain the fractional Riemann-Liouville.

2. Under the above conditions, if $k \neq 1$ then from Definition 2.1, the $k$-fractionals operators proposed in [18] are obtained.

3. If $\psi^{\prime}(t)=\frac{1}{t}, k=1$ and $G(z, \mu)=z^{1-\mu}$ then the Hadamard fractional operator (see [25]) is reproduced.

4. If $\psi^{\prime}(t)=\frac{1}{t^{\rho}}, G(z, \mu)=z^{1-\mu}$ and $k=1$, then we obtain the Katugampola's fractional operator defined in [14].

5. By choosing $k=1, G(z, \mu)=z^{1-\mu}$, we get the integral operator proposed in [15].

6. By taking $\psi^{\prime}(t)=1, k=1$ and $G(x-t, \mu)=\lambda^{\mu} \exp \left[-\frac{\lambda-1}{\lambda}(x-t)\right](x-t)^{1-\mu}$, we obtain the integral operator introduced in [20].

7. By choosing $k=1$ and $G(\psi(x)-\psi(t), \mu)=\frac{\lambda^{\mu}(\psi(x)-\psi(t))^{1-\mu}}{\exp \left[\frac{\lambda-1}{\lambda}(\psi(x)-\psi(t))\right]}$, we obtain the integral operator proposed in [22].

Next, we study some of the important properties of the operators specified in Definition 2.1. One of the basic questions that need to be answer for any newly defined integral operator is concerned about the boundedness.

Theorem 2.1. Let $h:\left[a_{1}, a_{2}\right] \rightarrow \mathbb{R}$ be an integrable function, and take $\mu>0$ and $k>0$. Then $I_{G, a_{1}+}^{\mu, k ;} h(x)$ exists for all $x \in\left[a_{1}, a_{2}\right]$.

Proof. Let $D=\left[a_{1}, a_{2}\right] \times\left[a_{1}, a_{2}\right]$ and define $C: D \rightarrow \mathbb{R}$ as

$$
C(x, t)=\left\{\begin{array}{cc}
\frac{\psi^{\prime}(t)}{G\left(\psi(x)-\psi(t), \frac{\mu}{k}\right)}, & a_{1} \leq t \leq x \leq a_{2}, \\
0, & a_{1} \leq x \leq t \leq a_{2} .
\end{array}\right.
$$

Taking into account that $C(x, t)$ is measurable on $D$ (the functions $\psi^{\prime}(t) \in A C\left[a_{1}, a_{2}\right]$ and $G(z, \mu) \in A C\left[a_{1}, a_{2}\right]$, on the compact $D$ ), we have

$$
I_{G, a_{1}+}^{\mu, k ; \psi} h(b)=I_{G, a_{1}+}^{\mu, k ; \psi} h(x)+I_{G, a_{2}-}^{\mu, k ; \psi} h(x)=\int_{a_{1}}^{a_{2}} C(x, s) h(s) d s=B(x) .
$$

If we take

$$
B=\sup _{\left[a_{1}, a_{2}\right]} B(x),
$$


then by using the the Tonelli's theorem for iterated integrals (see [8]), we have

$$
\begin{aligned}
\int_{a_{1}}^{a_{2}}\left[\int_{a_{1}}^{a_{2}} C(t, x)|h(t)| d t\right] d x & =\int_{a_{1}}^{a_{2}}|h(t)|\left[\int_{a_{1}}^{a_{2}} C(t, x) d t\right] d x \\
& \leq B\|h\|_{L_{1}\left[a_{1}, a_{2}\right]} \\
& <+\infty .
\end{aligned}
$$

Therefore, the function $E: D \rightarrow \mathbb{R}$, defined by $E(x, t)=C(x, t) h(x)$ is integrable over $D$.

Now using the Fubini's theorem, it follows that $\int_{a_{1}}^{a_{2}} C(t, x) h(t) d t$, as a function of $x \in\left[a_{1}, a_{2}\right]$, is integrable on $\left[a_{1}, a_{2}\right]$. This implies that $I_{G, a_{1}+}^{\mu, k ; \psi} h(x)$ is integrable on $\left[a_{1}, a_{2}\right]$.

Given the generality of the operator presented, what we can say is that if the function $G$ is additive with respect to the second variable, then it satisfies the Law of Semigroups.

Remark 2.2. Obviously, it can be thought that the additive property of the function $G$ is very restrictive. However, we draw our attention to the fact that all the operators presented above satisfy this certain property, but the generality of $G$ in our definition makes possible the existence of the functions that do not satisfy it.

Next, we give a result about the continuity of the operator given in Definition 2.1 .

Theorem 2.2. Let $k>0$ and $h \in L_{1}[a, b]$. Then $I_{G, a_{1}+}^{\mu, k ; \psi} h \in C[a, b]$.

Proof. Let $x, \xi \in[a, b], x \leq \xi$ and $x \rightarrow \xi$. Then, we have

$$
\begin{aligned}
& \left|I_{G, a_{1}+}^{\mu, k ; \psi} h(x)-I_{G, a_{1}+}^{\mu, k ; \psi} h(\xi)\right| \\
= & \frac{1}{k \Gamma_{k}(\mu)}\left|\int_{a_{1}}^{x} \frac{\psi^{\prime}(t)}{G\left(\psi(x)-\psi(t), \frac{\mu}{k}\right)} h(t) d t-\int_{a_{1}}^{\xi} \frac{\psi^{\prime}(t)}{G\left(\psi(\xi)-\psi(t), \frac{\mu}{k}\right)} h(t) d t\right| \\
= & \frac{1}{k \Gamma_{k}(\mu)}\left|\int_{a_{1}}^{x} \frac{\psi^{\prime}(t)}{G\left(\psi(x)-\psi(t), \frac{\mu}{k}\right)} h(t) d t-\int_{a_{1}}^{x} \frac{\psi^{\prime}(t)}{G\left(\psi(\xi)-\psi(t), \frac{\mu}{k}\right)} h(t) d t-\int_{x}^{\xi} \frac{\psi^{\prime}(t)}{G\left(\psi(\xi)-\psi(t), \frac{\mu}{k}\right)} h(t) d t\right| \\
= & \frac{1}{k \Gamma_{k}(\mu)}\left|\int_{a_{1}}^{x} \psi^{\prime}(t)\left(\frac{1}{G\left(\psi(x)-\psi(t), \frac{\mu}{k}\right)}-\frac{1}{G\left(\psi(\xi)-\psi(t), \frac{\mu}{k}\right)}\right) h(t) d t-\int_{x}^{\xi} \frac{\psi^{\prime}(t)}{G\left(\psi(\xi)-\psi(t), \frac{\mu}{k}\right)} h(t) d t\right| \\
\leq & \frac{1}{k \Gamma_{k}(\mu)} \int_{a_{1}}^{x}\left|\psi^{\prime}(t) h(t)\right|\left|\frac{1}{G\left(\psi(x)-\psi(t), \frac{\mu}{k}\right)}-\frac{1}{G\left(\psi(\xi)-\psi(t), \frac{\mu}{k}\right)}\right| d t \\
& +\frac{1}{k \Gamma_{k}(\mu)} \int_{x}^{\xi}\left|\psi^{\prime}(t) h(t)\right| \frac{1}{G\left(\psi(\xi)-\psi(t), \frac{\mu}{k}\right)} \mid d t .
\end{aligned}
$$

Therefore, by dominated convergence theorem, we obtain

$$
\left|I_{G, a_{1}+}^{\mu, k ; \psi} h(x)-I_{G, a_{1}+}^{\mu, k ; \psi} h(\xi)\right| \rightarrow 0
$$

as $x \rightarrow \xi$, that is,

$$
I_{G, a_{1}+}^{\mu, k ; \psi} h \in C[a, b]
$$

\section{A particular case}

Many natural processes, growth and learning curves of complex systems, show a temporal progression from low levels at the beginning, approach a climax after a certain time; the transition occurs in a region characterized by strong intermediate acceleration. The sigmoid function, a continuous non-linear function, allows us to describe this evolution. Sometimes the sigmoid function is called logistic function (see [9, 17, 26,30]). The sigmoid function is defined as $\sigma(x)=\frac{1}{1+e^{-x}}$, and its derivative is $\sigma^{\prime}(x)=\sigma(x)(1-\sigma(x))$. By taking $k=1, G(z, \mu)=z^{1-\mu}$ and $\psi(u)=\sigma(u)$ in Definition 2.1, we obtain (see $[16,23])$ :

$$
I_{a+}^{\mu ; \sigma} f(x)=\int_{a}^{x} \frac{\sigma(t)(1-\sigma(t)) f(t)}{(\sigma(x)-\sigma(t))^{1-\mu}} d t
$$


and

$$
{ }^{H} D_{a+}^{\mu, \beta} f(x)=I_{a+}^{\beta(n-\mu) ; \sigma}\left(\frac{1}{\sigma^{\prime}(x)} \frac{d}{d x}\right)^{n} I_{a+}^{(1-\beta)(n-\mu) ; \sigma} f(x) .
$$

These operators have been proven useful in various practical problems (from the population growth to the shallow water wave and reaction-diffusion process). We end this paper by defining the following generalized sigmoid function (which may open a new line of research):

$$
\sigma(x)=\frac{1}{1+e^{-g(x)}},
$$

where $g(x)$ is a smooth function.

\section{Acknowledgment}

The author wish to thank the anonymous reviewers for their suggestions and comments, which have improved the quality of this paper.

\section{References}

[1] A. Atangana, Derivative with a New Parameter: Theory, Methods and Applications, Academic Press, London, 2016.

[2] D. Baleanu, Comments on: "The failure of certain fractional calculus operators in two physical models" by M. Ortigueira, V. Martynyuk, M. Fedula and J.A.T. Machado, Fract. Calc. Appl. Anal. 23 (2020) 292-297.

[3] D. Baleanu, A. Fernandez, On fractional operators and their classifications, Mathematics 7 (2019) Art\# 830.

[4] M. Caputo, Elasticità e Dissipazione, Bologna, Zanichelli, 1969.

[5] M. Caputo, Linear model of dissipation whose Q is almost frequency independent II, Geophys. J. Int. 13 (1967) 529-539.

[6] R. Díaz, E. Pariguan, On hypergeometric functions and Pochhammer k-symbol, Divulg. Mat. 15 (2007) 179-192.

[7] P. M. Guzmán, L. M. Lugo Motta, J. E. Nápoles Valdés, M. Vivas-Cortez, On a new generalized integral operator and certain operating properties, Axioms 9 (2020) Art\# 69.

[8] P. R. Halmos, Measure Theory, D. Van Nostrand Company, New York, 1950.

[9] N. Hassan, N. Akamatsu, A new approach for contrast enhancement using sigmoid function, Int. Arab J. Inf. Tech. 1 (2004) $221-225$.

[10] R. Hilfer, Experimental evidence for fractional time evolution in glass forming materials, Chem. Phys. 284 (2002) $399-408$.

[11] R. Hilfer, Fractional calculus and regular variation in thermodynamics, In: R. Hilfer (Ed.), Applications of Fractional Calculus in Physics, World Scientific, Singapore, 2000, pp. 429-463.

[12] R. Hilfer, Fractional time evolution, In: R. Hilfer (Ed.), Applications of Fractional Calculus in Physics, World Scientific, Singapore, 2000, pp. 87-130.

[13] R. Hilfer, Y. Luchko, Z. Tomovski, Operational method for the solution of fractional differential equations with generalized Riemann-Liouville fractional derivatives, Frac. Calc. Appl. Anal. 12 (2009) 299-318.

[14] U. N. Katugampola, New approach generalized fractional integral, Appl. Math. Comput. 218 (2011) 860-865.

[15] A. A. Kilbas, H. M. Srivastava, J. J. Trujillo, Theory and Applications of Fractional Differential Equations, Elsevier, New York, 2006.

[16] J. G. Liu, X. J. Yang, Y. Y. Feng, P. Cui, New fractional derivative with sigmoid function as the kernel and its models, Chinese J. Phys. 68 (2020) $533-541$.

[17] A. C. Marreiros, J. Daunizeau, S. J. Kiebel, K. J. Friston, Population dynamics: variance and the sigmoid activation function, Neuroimage 42 (2008) $147-157$.

[18] S. Mubeen, G. M. Habibullah, $k$-fractional integrals and applications, Int. J. Contemp. Math. Sci. 7 (2012) 89-94.

[19] J. E. Nápoles Valdés, P. M. Guzmán, L. M. Lugo Motta, A. Kashuri, The local generalized derivative and Mittag Leffler function, Sigma J. Eng. Nat. Sci. 38 (2020) 1007-1017.

[20] G. Rahman, T. Abdeljawad, F. Jarad, A. Khan, K. S. Nisar, Certain inequalities via generalized proportional Hadamard fractional integral operators, Adv. Difference Equ. 2019 (2019) Art\# 454.

[21] E. D. Rainville, Special Functions, Macmillan Co., New York, 1960.

[22] S. Rashid, F. Jarad, M. A. Noor, H. Kalsoom, Y. M. Chu, Inequalities by means of generalized proportional fractional integral operators with respect to another function, Mathematics 7 (2020) Art\# 1225.

[23] M. Rezapour, A. Sijuwade, T. Asaki, A sigmoidal fractional derivative for regularization, AIMS Math. 5 3284-3297.

[24] F. Qi, B. N. Guo, Integral representations and complete monotonicity of remainders of the Binet and Stirling formulas for the gamma function, Rev. R. Acad. Cienc. Exactas Fís. Nat. Ser. A Mat. 111 (2017) 425-434.

[25] S. G. Samko, A. A. Kilbas, O. I. Marichev, Fractional Integrals and Derivatives: Theory and Applications, Gordon \& Breach, Yverdon, 1993.

[26] M. T. Tommiska, Efficient digital implementation of the sigmoid function for reprogrammable logic, IEE Proc. - Comput. Digit. Tech. 150 (2003) $403-411$.

[27] S. Umarov, S. Steinberg, Variable order differential equations with piecewise constant order-function and diffusion with changing modes, $Z$. Anal. Anwend. 28 (2009) 431-450.

[28] Z. H. Yang, J. F. Tian, Monotonicity and inequalities for the gamma function. J. Inequal. Appl. 2017 (2017) Art\# 317.

[29] Z. H. Yang, J. F. Tian, Monotonicity and sharp inequalities related to gamma function, J. Math. Inequal. 12 (2018) 1-22.

[30] P. C. Yong, S. Nordholm, H. H. Dam, Optimization and evaluation of sigmoid function with a priori SNR estimate for real-time speech enhancement, Speech Commun. 55 (2013) 358-376.

[31] D. Zhao, M. Luo, General conformable fractional derivative and its physical interpretation, Calcolo 54 (2017) $903-917$. 